\title{
Regulation of antioxidant enzyme activities in male and female rat macrophages by sex steroids
}

\section{R.B. Azevedo ${ }^{1}$, \\ Z.G.M. Lacava ${ }^{1}$, \\ C.K. Miyasaka², \\ S.B. Chaves ${ }^{1}$ and R. Curi}

\author{
1Departamento de Genética e Morfologia, Instituto de Ciências Biológicas, \\ Universidade de Brasília, Brasília, DF, Brasil \\ 2Departamento de Fisiologia e Biofísica, Instituto de Ciências Biológicas, \\ Universidade de São Paulo, São Paulo, SP, Brasil
}

\section{Correspondence \\ R.B. Azevedo \\ Departamento de Genética e \\ Morfologia, ICB, UnB \\ 70910-900 Brasília, DF \\ Brasil \\ Research supported by FAPESP,} CAPES, CNPq, Pronex and FAP-DF.

Received August 8, 2000 Accepted February 13, 2001

\section{Abstract}

Human and animal immune functions present sex dimorphism that seems to be mainly regulated by sex hormones. In the present study, the activities of the antioxidant enzymes total superoxide dismutase (SOD), catalase (CAT), and glutathione peroxidase (GSH-Px) were measured in intraperitoneal resident macrophages from adult male and female rats. In addition to comparing males and females, we also examined the regulation of these enzyme activities in macrophages by sex steroids. GSH-Px activity did not differ between male and female macrophages. However, both total SOD and CAT activities were markedly higher in females than in males ( 83 and 180\%). Removal of the gonads in both males and females (comparison between castrated groups) increased the difference in SOD activity from 83 to $138 \%$ and reduced the difference in CAT activity from 180 to $86 \%$. Castration and testosterone administration did not significantly modify the activities of the antioxidant enzymes in male macrophages. Ovariectomy did not affect SOD or GSH-Px activity but markedly reduced (48\%) CAT activity. This latter change was fully reversed by estrogen administration, whereas progesterone had a smaller effect. These results led us to conclude that differences in the SOD and CAT activities may partially explain some of the differences in immune function reported for males and females. Also, estrogen is a potent regulator of CAT in macrophages and therefore this enzyme activity in macrophages may vary considerably during the menstrual cycle.

\section{Key words}

- Antioxidant enzymes

- Macrophages

- Sex hormones

- Estrogen

- Progesterone

- Castration
Several aspects of both the humoral and cellular immune responses differ between males and females $(1,2)$ and this dimorphism is mainly caused by sex steroid hormones (3). Specific receptors for gonadal steroids have been found in the lymphoid organs and macrophages (1). Macrophages play a central role in the inflammatory and immune responses (4). These cells are able to kill foreign organisms and expose antigens on the surface of the membrane for the immune response to be initiated by lymphocytes. Macrophages present high endocytic activity and capacity to produce a number of multifunctional compounds including cytokines and reactive oxygen species (ROS), such as superoxide anion $\left(\mathrm{O}_{2}^{-}\right)$, hydrogen peroxide $\left(\mathrm{H}_{2} \mathrm{O}_{2}\right)$ and hydroxyl radical $\left(\mathrm{OH}^{\bullet}\right)(5)$. These ROS are known to cause DNA damage and lipid peroxidation/oxidation in the mem- 
branes (6). To protect themselves against the adverse effects of the ROS, these cells present a complex machinery of antioxidant compounds and enzymes, such as superoxide dismutase (SOD), catalase (CAT), and glutathione peroxidase (GSH-Px) (7). The activities of these enzymes have been shown to be regulated by nutrients $(6,7)$ and hormones (8).

The role of sex hormones in lipid peroxidation has been investigated in rat liver homogenates. Male rats have a higher content of products of lipid peroxidation than females (9). There is substantial evidence that estrogen presents antioxidant properties $(10,11)$. The antioxidant effect of estrogen has been regarded as the main mechanism for this hormone to protect skeletal and cardiac muscles (12), uterus (13), and liver (9) from damage. In brain, progesterone instead of estrogen exerts a significant antioxidant effect (14). Contrary to female steroids, testosterone has been shown to decrease the activities of SOD, CAT, and GSH-Px, leading to lipid peroxidation (15).

On the basis of these considerations, we investigated the possible role of gonadal steroids in the protection against oxidative stress in macrophages. We compared the activities of the antioxidant enzymes (SOD, CAT, and GSH-Px) in female and male rat macrophages and examined the effect of gonadectomy and the sex steroid hormone replacement in both male and female rats.

Male and female albino Wistar rats, 3 months old, were obtained from the Institute of Biomedical Sciences, São Paulo, SP, Brazil. The rats were kept at $23^{\circ} \mathrm{C}$ on a light/dark cycle of 12/12 h (lights on from 7:00 am). All chemicals and enzymes were obtained from Boehringer-Mannheim GmbH (Mannheim, Germany), or Sigma Chemical Co. (St. Louis, MO, USA). The sex steroid hormones were obtained from Sigma. Male and female rats were gonadectomized under ether anesthesia. After one month, the rats were decapitated without anesthesia between 10:00 and
12:00 am. Some of the castrated animals were treated with steroid hormones as follows: male castrated animals received $10 \mu \mathrm{g}$ testosterone daily and ovariectomized rats received $200 \mathrm{ng}$ estrogen or $1 \mu \mathrm{g}$ progesterone, or both, daily. The hormones were injected subcutaneously for 4 days before obtaining the cells. The control group was treated with saline. The rats were killed $4 \mathrm{~h}$ after the last injection. The protocol of sex steroid hormone replacement was the same as used in a previous study by our group (2).

Cells normally present in the intraperitoneal cavity of the rats were collected using 6 $\mathrm{ml}$ PBS, and allowed to adhere to glass flasks for $2 \mathrm{~h}$ at $37^{\circ} \mathrm{C}$, after which time the medium and nonadherent cells were removed, and the remaining cells were called resident macrophages (unstimulated). Cell viability was determined by Trypan blue exclusion $(>95 \%)$. At least $92 \%$ of the cells were macrophages as determined by differential counts. The macrophages were homogenized with a polytron apparatus (PCU-2) at a proportion of 1:10 in $10 \mathrm{mM}$ sodium phosphate buffer, $\mathrm{pH}$ 7.5. CAT activity was determined by monitoring hydrogen peroxide consumption at $230 \mathrm{~nm}$ and $30^{\circ} \mathrm{C}(16)$. GSH-Px activity was measured as described by Wendel (17), by monitoring the decrease of NADPH concentration at $340 \mathrm{~nm}$ and $37^{\circ} \mathrm{C}$. Total SOD activity (16) was measured on the basis of the rate of cytochrome $c$ reduction by $\mathrm{O}_{2}^{-\bullet}$ monitored at $550 \mathrm{~nm}$ and $25^{\circ} \mathrm{C}$ using the xanthine-xanthine-oxidase system as the source of $\mathrm{O}_{2}^{-\bullet}$. SOD competes for $\mathrm{O}_{2}^{-\bullet}$ and decreases the rate of cytochrome $c$ reduction. All measurements were performed with a Gilford recording spectrophotometer (model Response). CAT and SOD activities are reported as $\mu \mathrm{mol}$ per minute per mg protein (units per mg protein), and GSH-Px activity is reported as nmol per minute per mg protein. Data are reported as the mean $\pm \mathrm{SEM}$. The $t$-test was employed for comparisons between female and male control groups, and between female and male castrated 
groups. ANOVA and Duncan's test were used for comparison between treatments within the same group. Differences were considered to be significant at $\mathrm{P}<0.05$.

GSH-Px activity did not differ between male and female macrophages (see Tables 1 and 2 for comparison). However, both total SOD and CAT activities were markedly higher in females than in males (83 and $180 \%$, respectively). Removal of the gonads in both males and females (comparison between castrated groups) raised the difference in SOD activity to $138 \%$ and reduced the difference in CAT activity to $86 \%$. Nevertheless, the female rats always showed higher antioxidant enzyme activity. Even gonadectomized females presented higher CAT and SOD activities than the control male rats. One may then conclude that the capacity of female macrophages to protect themselves against oxidative stress is really higher than that observed in male cells. This might be an important mechanism for the high immunoreactivity described for females $(1,18)$.

Administration of testosterone has been shown to increase lipid peroxidation in rat testis (15). This effect is accompanied by a significant decrease in SOD, CAT, and GSHPx activities. In this study, however, castration and testosterone replacement did not significantly affect the activities of the antioxidant enzymes in male macrophages. Therefore, the pro-oxidant effect of testosterone may be restricted to only a few tissues.

Ovariectomy did not affect SOD or GSHPx activity but reduced CAT activity by $48 \%$ (Table 1). This change was fully reversed by estrogen administration whereas progesterone had a smaller effect (Table 1). On the other hand, the CAT activity of macrophages from rats treated with estrogen plus progesterone did not differ from that of the groups which received only estrogen. Therefore, it is very unlikely that the two hormones present a synergistic effect on the regulation of CAT activity (Table 1). Since estrogen is a potent regulator of CAT in macrophages, this enzyme activity may vary considerably during the menstrual cycle. Whether this fact has significant implications for macrophage function and thus for the immune and inflammatory responses remains to be investigated.

Previous studies have shown that estrogen affects several aspects of macrophage function. This hormone increases the expression of surface markers and the production of interleukin-1 (19). Suppression of gonadal steroids by ovariectomy reduces

Table 1. Effect of castration and hormone replacement on catalase (CAT), total superoxide dismutase (SOD) and glutathione peroxidase (GSH-PX) specific activity of peritoneal macrophages from female rats.

\begin{tabular}{lccccc}
\hline Enzymes & \multicolumn{5}{c}{ Groups } \\
\cline { 2 - 6 } & Control & Castrated & Estrogen & Progesterone & $\begin{array}{c}\text { Estrogen }+ \\
\text { progesterone }\end{array}$ \\
\hline CAT & $19.9 \pm 2.2$ & $10.4 \pm 1.1^{*}$ & $24.4 \pm 2.0$ & $15.3 \pm 1.8^{*}$ & $23.6 \pm 2.8$ \\
SOD & $576 \pm 60$ & $675 \pm 71$ & $600 \pm 80$ & $599 \pm 40$ & $622 \pm 77$ \\
GSH-Px & $0.74 \pm 0.05$ & $0.81 \pm 0.08$ & $0.80 \pm 0.08$ & $0.79 \pm 0.07$ & $0.70 \pm 0.07$
\end{tabular}

Specific activities are expressed as $\mathrm{U} / \mathrm{mg}$ protein. Data are reported as means $\pm \mathrm{SEM}$ for 8 rats. $* \mathrm{P}<0.05$ compared to control group (ANOVA and Duncan test). For details of macrophage preparation and hormone administration, see text.

Table 2. Effect of castration and testosterone replacement on catalase (CAT), total superoxide dismutase (SOD) and glutathione peroxidase (GSH$\mathrm{Px}$ ) specific activity of peritoneal macrophages from male rats.

\begin{tabular}{lccc}
\hline \multirow{3}{*}{ Enzymes } & \multicolumn{3}{c}{ Groups } \\
\cline { 2 - 4 } & Control & Castrated & Testosterone \\
\hline CAT & $7.1 \pm 0.6$ & $5.6 \pm 0.6$ & $5.5 \pm 0.48$ \\
SOD & $314 \pm 23$ & $283 \pm 33$ & $295 \pm 30$ \\
GSH-Px & $0.82 \pm 0.09$ & $0.66 \pm 0.08$ & $0.62 \pm 0.08$
\end{tabular}

Specific activities are expressed as $\mathrm{U} / \mathrm{mg}$ protein. Data are reported as means \pm SEM for 5-8 rats. There was no significant effect of either castration or hormone replacement. The CAT and SOD activities of male control and castrated rats were significantly lower than those of the corresponding female rats (Table 1$)(P<0.05$, Student t-test). For details of macrophage preparation and hormone administration, see text. 
hydrogen peroxide production and phagocytic capacity by rat macrophages and the changes are abolished by estrogen treatment (2). Lacava and Luna (11) showed that ovariectomy induces a significant increase in the frequency of structural chromosome aberrations in peritoneal macrophages, which is also reversed by estrogen administration. These authors suggested the involvement of ROS in the chromosome damage caused by the lack of gonadal steroids.

Sex steroid hormones regulate the activities of a number of enzymes of the glucose and glutamine metabolism in leukocytes. Azevedo et al. (2) showed that the activity of phosphate-dependent glutaminase is regulated by estrogen in rat peritoneal macrophages. In the same study, the authors found a decrease in the phagocytic capacity and hydrogen peroxide production of macrophages from castrated female rats. These changes were all reversed by estrogen treatment. In the present study evidence is presented that in addition to the metabolic en- zymes, estrogen also controls the activity of the antioxidant enzyme CAT. Changes in the oxidative defense system do impair macrophage function $(4,8)$. Then, the control of macrophage function by estrogen may involve several mechanisms including cell metabolism and oxidative stress.

The findings presented herein led us to conclude that differences in the enzymatic antioxidant capacity (mainly CAT and SOD) between male and female macrophages may partially explain the dimorphism widely reported for the immune function. Also, the role played by the female sex steroids in the high immunoreactivity observed in females may occur by modulation of the antioxidant defense system. Additional studies are needed to further address this important issue.

\section{Acknowledgments}

The authors are indebted to J.R. Mendonça and G. de Souza for technical assistance.

\section{References}

1. Grossman CJ (1985). Interactions between the gonadal steroids and immune system. Science, 227: 257-261.

2. Azevedo RB, Costa-Rosa LFBP, Lacava ZGM \& Curi R (1997). Gonadectomy impairs lymphocyte proliferation and macrophage function in male and female rats. Correlation with key enzyme activities of glucose and glutamine metabolism. Cell Biochemistry and Function, 15: 293-298.

3. Ansar-Ahmed S, Penhale WJ \& Talal N (1985). Sex hormones in immune responses and autoimmune diseases. American J ournal of Pathology, 121: 531551.

4. Newsholme P, Costa-Rosa LFBP \& Curi R (1996). The importance of macrophage fuel metabolism to its function. Cell Biochemistry and Function, 14: 1-10.

5. Curi R, Newsholme P, Pithon-Curi TC, Pires-de-Melo M, Garcia C, Homem-deBittencourtJ r PI \& Guimarães ARP (1999). Metabolic fate of glutamine in lympho- cytes, macrophages and neutrophils. Brazilian J ournal of Medical and Biological Research, 32: 15-21.

6. Miyasaka CK, De-Souza J AA, Torres RP, Mancini-Filho J, Lajolo FM \& Curi R (1998). Effect of the administration of fish oil by gavage on activities of antioxidant enzymes of rat lymphoid organs. General Pharmacology, 30: 759-762.

7. Harris ED (1992). Regulation of antioxidant enzymes. J ournal of Nutrition, 122: 625-626.

8. Pereira B, Costa-Rosa LFBP, Bechara EJ H, Newsholme P \& Curi R (1998). Changes in TBARS content and superoxide dismutase, catalase and glutathione peroxidase activities in the lymphoid organs and skeletal muscles of adrenodemedullated rats. Brazilian J ournal of Medical and Biological Research, 31: 827-833.

9. Huh K, Shin US, Choi J W \& Lee SI (1994). Effect of sex hormone on lipid peroxidation in liver. Archives of Pharmacal Re- search, 17: 109-114.

10. Gomez-Zubeldia MA, Hernandez R, Viguera J, Arbues JJ, Aparicio A \& Millan JC (2000). Effect of bilateral ovariectomy and ovarian steroid hormones on the antioxidant systems and plasma malondialdehyde levels in Wistar rats. Endocrine Research, 26: 97-107.

11. Lacava ZGM \& Luna H (1994). The anticlastogenic effect of tocopherol in peritoneal macrophages of benznidazoletreated and ovariectomized mice. Mutation Research, 305: 145-150.

12. Persky AM, Green PS, Stubley L, Howell CO, Zaulyanov L, Brazeau GA \& Simpkins J W (2000). Protective effect of estrogens against oxidative damage to heart and skeletal muscle in vivo and in vitro. Proceedings of the Society for Experimental Biology and Medicine, 223: 59-66.

13. Diaz-Flores M, Baiza-Gutman LA, Pedron NN \& Hicks J J (1999). Uterine glutathione reductase activity: modulation by estro- 
gens and progesterone. Life Sciences, 65: 2481-2488.

14. Pajovic SB, Saicic ZS, Spasic MB, Petrovic VM \& Martinovic J V (1999). Effects of progesterone and estradiol benzoate on glutathione dependent antioxidant enzyme activities in the brain of female rats. General Physiology and Biophysics, 18: 35-44.
15. Chainy GB, Samantaray S \& Samanta L (1997). Testosterone-induced changes in testicular antioxidant system. Andrologia, 29: 343-349.

16. Flohé $L$ \& Ötting F (1984). Superoxide dismutase assays. Methods in Enzymology, 105: 93-104.

17. Wendel A (1981). Glutathione peroxidase. Methods in Enzymology, 77: 325-339.
18. Kimura M, Watanable $H$, Sato $S \& A b o ~ T$ (1994). Female predominance of extrathymic $T$ cells in mice: statistical analysis. Immunology Letters, 39: 259-267.

19. Flynn A (1986). Expression of la and the production of interleukin-1 by peritoneal exudate macrophages activated in vivo by steroids. Life Sciences, 38: 2455-2460. 